\title{
CAS 2.0: Evolução e Automação do Checklist de Avaliação do Scrum para Projetos de Software
}

\author{
Thiago F. V. da Cunha ${ }^{1,3}$, Ismayle de S. Santos, ${ }^{2,3, a}$, Alysson A. de Macedo ${ }^{2,3}$, Alberto \\ H. M. T. Monteiro ${ }^{3}$, Bruno S. Aragão ${ }^{2,3}$, Rossana M. C. Andrade ${ }^{2,3, b}$ \\ ${ }^{1}$ Dataprev \\ Fortaleza, Ceará, Brasil \\ ${ }^{2}$ Departamento de Computação - Universidade Federal do Ceará (UFC) \\ Fortaleza, Ceará, Brasil \\ ${ }^{3}$ Grupo de Redes de Computadores, Engenharia de Software e Sistemas (GREat) \\ Fortaleza, Ceará, Brasil \\ \{thiagoferraz, ismaylesantos, alyssonmacedo, bruno, rossana\} egreat.ufc.br
}

Resumo. Este trabalho propõe uma evolução e automação de um checklist já existente, denominado Checklist de Avaliação do Scrum (CAS), componente do Agile DMAIC, que é um método para avaliar e melhorar o uso do Scrum em projetos de software. Com o CAS, e possível avaliar a abrangência do Scrum nos projetos e identificar melhorias. A evolução apresentada neste artigo visa ampliar a inspeção de princípios e práticas ágeis, e foi realizada de forma sistemática. A automação possibilita que o CAS consolide a visão das equipes sobre o Scrum e forneça resultados em tempo real, a partir de um preenchimento online. Para avaliar o CAS 2.0, ele foi aplicado em quinze projetos reais e seus resultados são também discutidos neste artigo.

Abstract. This paper proposes an evolution and automation of an existing checklist, called Scrum Assessment Checklist (CAS), component of Agile DMAIC, which is a method to evaluate and improve the use of Scrum in software projects. With CAS, one can assess the Scrum coverage in projects and identify improvements. The evolution presented in this article aims to increase the inspection of agile principles and practices, and was carried out systematically. The automation enables the CAS to consolidate teams' vision about the Scrum and provide real-time results from an online filling. To evaluate the CAS 2.0, it was applied in fifteen real projects and their results are also discussed in this article.

\section{Introdução}

As metodologias ágeis objetivam acelerar o desenvolvimento de software a partir da melhoria contínua dos processos, gerando benefícios na comunicação e interação da equipe, organização, diminuição de falhas, respostas mais rápidas às mudanças e aumento da produtividade do time de desenvolvimento [Schwaber 2007].

\footnotetext{
${ }^{a}$ Bolsista de doutorado da CAPES

${ }^{\mathrm{b}}$ Bolsista do CNPq de Produtividade em Desenvolvimento Tecnológico e Extensão Inovadora (DT) 2
} 
O Scrum [Sutherland e Schwaber 2016] é uma das metodologias ágeis mais utilizadas no desenvolvimento de software e tem contribuído na melhoria da gestão dos projetos, aumentando a probabilidade de sucesso das equipes [VersionOne 2016]. Criada em 1996, essa metodologia adota uma abordagem empírica e pode ser utilizada em projetos complexos com, por exemplo, uma grande quantidade de produtos diferentes, uso simultâneo de várias tecnologias, muitas restrições tecnológicas e incertezas associadas [Melo et al. 2012]. Entretanto, para que os projetos usufruam dos principais benefícios do Scrum, como a melhoria da produtividade e qualidade dos produtos desenvolvidos, ele precisa ser adequadamente instanciado de acordo com o contexto de cada projeto, organização e cliente. Além disso, essa instanciação precisa evoluir (e.g., revisar e ajustar os tamanhos da equipe e do sprint, os papéis dos envolvidos, a forma como a equipe realiza os eventos e utiliza os artefatos do Scrum) durante a execução dos projetos. Surge, portanto, a necessidade de gerenciar a adoção ou uso do Scrum para a evolução contínua e controlada do processo ágil.

Nesse cenário, o Agile DMAIC [Cunha e Andrade 2014a] é um método que possibilita às equipes gerenciarem a adoção ou uso do Scrum, promovendo uma gestão quantitativa do uso de seus princípios e práticas, bem como a identificação e o tratamento de causas de problemas para a melhoria contínua da agilidade.

Um dos componentes do Agile DMAIC é o Checklist de Avaliação do Scrum $(C A S)$, utilizado para coletar a percepção das equipes sobre o uso do Scrum. Sua primeira versão, elaborada com base apenas no Nokia Test [Vode e Sutherland 2008] e no Scrum Checklist [Kniberg 2009], não abrange algumas práticas importantes do Scrum (e.g., definição dos objetivos do sprint, revisão e atualização da definição de "pronto", atenção do time à qualidade do software desenvolvido) que estão no Guia do Scrum [Sutherland e Schwaber 2016], uma das principais referências sobre essa metodologia. Além disso, para facilitar o reuso do $C A S$, ele precisa ser automatizado, permitindo o seu preenchimento online.

Este trabalho propõe, então, uma evolução e automação do Checklist de Avaliação do Scrum (CAS) [Cunha e Andrade 2014a], componente do Agile DMAIC. Essa evolução visa ampliar a inspeção de princípios e práticas ágeis, e foi realizada de forma sistemática, considerando o Guia do Scrum e outros sete checklists de avaliação do uso do Scrum. A automação possibilita que o $C A S$ consolide a visão das equipes sobre o Scrum e forneça resultados em tempo real, a partir de um preenchimento online. Para avaliar a nova versão do $C A S$, ela foi aplicada em quinze projetos reais e seus resultados, que também são discutidos neste artigo, foram positivos.

Para apresentar o CAS 2.0, este artigo está estruturado em mais seis seções. Os trabalhos relacionados ao uso de checklists de avaliação do Scrum são discutidos na Seção 2. O Agile DMAIC é apresentado na Seção 3 e a Seção 4 descreve o processo de elaboração do novo checklist. A Seção 5 apresenta o CAS 2.0, detalhando o seu objetivo, público alvo, conteúdo e a forma adequada de sua utilização, bem como um comparativo das versões desse checklist e a arquitetura de sua automação. Os resultados da aplicação do $C A S 2.0$ em projetos reais são discutidos na Seção 6 e, finalmente, na Seção 7, são apresentadas as conclusões e trabalhos futuros. 


\section{Trabalhos Relacionados}

Para encontrar os trabalhos relacionados foi realizada uma busca na literatura por publicações sobre os seguintes tópicos: (i) checklists de avaliação do Scrum; e (ii) conformidade de processos de desenvolvimento de software. Como locais de busca, foram utilizadas as bases ACM DL Library ${ }^{1}$,Web of Science $^{2}$, Scopus $^{3}$ e os anais do Simpósio Brasileiro de Qualidade de Software (SBQS). Adicionalmente, o Google $\mathrm{e}^{4}$ Google Scholar ${ }^{5}$.

A maioria das ferramentas de avaliação de processos ágeis fornece um checklist de avaliação e uma análise das respostas de seu preenchimento, e foca em uma ou mais metodologias ágeis [Jalali et al. 2014]. Nas buscas por trabalhos relacionados à avaliação do Scrum, foram identificados dois trabalhos [Park et al. 2016, Cunha e Andrade 2014a] que propõem métodos de avaliação do Scrum que utilizam checklists.

Cunha e Andrade [Cunha e Andrade 2014a] apresentam o Agile DMAIC (descrito na Seção 3), um método que possibilita avaliar e melhorar o uso do Scrum, fornecendo medições qualitativas e quantitativas de sua aplicação nos projetos. Para isso, os autores propõem a primeira versão do CAS, um checklist elaborado a partir do Nokia Test [Vode e Sutherland 2008] e do Scrum Checklist [Kniberg 2009]. Park et al. [Park et al. 2016] descrevem o Scrum através do Essence, um padrão para elaboração e melhoria de métodos de engenharia de software. Os autores propõem mapeamentos entre as categorias do Essence e os artefatos, eventos e papéis do Scrum. Através desses mapeamentos, são especificados checklists para avaliar o progresso dos projetos. Uma desvantagem é a necessidade de entendimento do Essence, mesmo que superficial. Além disso, apenas um dos checklists é apresentado (sobre o planejamento de releases).

Quanto aos checklists publicados em páginas online, foram identificados os seguintes: (i) checklist da firma de consultoria Total Programme Control [TPC 2016]; (ii) checklist de Maharana [Maharana 2015]; (iii) checklist de Lomonaco e Lapointe [Lomonaco e Lapointe 2013]; (iv) InfoQ Scrum Checklist [Gloger 2012]; (v) Scrum Checklist [Kniberg 2009]; e (vi) Nokia Test [Vode e Sutherland 2008].

O comparativo dos checklists supracitados é apresentado na Tabela 1. A maioria dos checklists possui uma baixa cobertura (abrangência) dos princípios e práticas do Scrum [TPC 2016], [Maharana 2015], [Lomonaco e Lapointe 2013], [Gloger 2012] e [Vode e Sutherland 2008]). Contudo, as maiores limitações dizem respeito a consolidação do preenchimento pela equipe e a apresentação de estudos de caso avaliando os checklists, identificados apenas no CAS [Cunha e Andrade 2014a], e a exibição de resultados em tempo real, implementada por apenas três checklists ([TPC 2016], [Lomonaco e Lapointe 2013], e [Vode e Sutherland 2008]).

Além disso, existem trabalhos que não apresentam um checklist, mas estão relacionados à detecção de problemas em processos. Zazworka et al. [Zazworka et al. 2010] apresentam uma abordagem genérica para detectar não conformidades com o processo, baseada em dados fornecidos pelo analista de conformidade do processo e

\footnotetext{
${ }^{1}$ http://dl.acm.org/

${ }^{2}$ https://webofknowledge.com/

${ }^{3}$ https://www.scopus.com/

${ }^{4}$ https://google.com/

${ }^{5}$ http://scholar.google.com.br/
} 
informações capturadas por um sistema de controle de versão. Khelladi et al. [Khelladi et al. 2015] apresentam um conjunto de sete restrições formalizando as melhores práticas do Scrum. Essas restrições podem ser verificadas em um modelo de processo utilizado. Perkusich et al. [Perkusich et al. 2015] apresentam um processo de avaliação da aplicação do Scrum com redes Bayesianas, que possuem como entradas métricas e evidências sobre o uso do Scrum. A compilação dessas entradas, na rede Bayesiana definida, permite identificar as deficiências para reflexão da equipe e definição de planos de ações durante as retrospectivas dos sprints.

Tabela 1. Comparativo dos Checklists

\begin{tabular}{|c|c|c|c|c|}
\hline Checklists & Abrangência & $\begin{array}{l}\text { Resultado em } \\
\text { Tempo Real }\end{array}$ & $\begin{array}{c}\text { Consolidação do } \\
\text { Preenchimento }\end{array}$ & Estudo de Caso \\
\hline TCP 2016 & $\mathbf{D}$ & 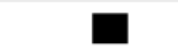 & $\square$ & $\square$ \\
\hline Maharana 2015 & $\mathbf{D}$ & $\square$ & $\square$ & $\square$ \\
\hline Cunha e Andrade 2014a (CAS) & & $\square$ & $\square$ & $\square$ \\
\hline Lomonaco e Lapointe 2013 & & 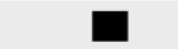 & $\square$ & $\square$ \\
\hline Gloger 2012 (InfoQ Scrum Checklist) & E & $\square$ & $\square$ & $\square$ \\
\hline Kninberg 2009 (Scrum Checklist) & & $\square$ & $\square$ & $\square$ \\
\hline Vode e Sutherland 2008 (Nokia Test) & $\square$ & $\square$ & $\square$ & $\square$ \\
\hline Atende & $\square$ Ate & parcialmente & $\square$ Não a & \\
\hline
\end{tabular}

As principais desvantagens dessas abordagens são a cobertura dos elementos do Scrum, que é baixa no trabalho de Khelladi et al., e o esforço e conhecimento adicional para realizar a verificação da conformidade com o processo, como é o caso dos trabalhos de Zazworka et al. e Perkusich et al..

Nesse contexto, este artigo aborda essas lacunas existentes, propondo o CAS 2.0, que é uma evolução do checklist apresentado em [Cunha e Andrade 2014a]. Essa nova versão foi elaborada de forma sistemática, considerando o Guia do Scrum e os checklists elencados nesta seção. O processo de elaboração do $C A S 2.0$, o CAS 2.0 e a avaliação de sua aplicação em projetos reais são apresentados e discutidos nas seções 4,5 e 6 , respectivamente.

\section{Agile DMAIC}

Agile DMAIC [Cunha e Andrade 2014a] é um método para avaliação e melhoria do uso de princípios e práticas do Scrum, que pode ser usado por equipes iniciantes ou experientes, na adoção e na melhoria da utilização dessa metodologia.

As principais contribuições do Agile DMAIC são: (i) possibilitar uma gestão quantitativa do uso do Scrum; (ii) avaliar o uso de seus princípios e práticas; e (iii) identificar e tratar as causas de problemas, considerando o contexto e a necessidade dos projetos e clientes.

O Agile DMAIC utiliza princípios e técnicas do Lean Six Sigma [Radujkovic et al. 2014] e foca na execução, de forma iterativa e incremental, de uma simplificação do ciclo de melhoria DMAIC. Essa execução é realizada em paralelo ao uso do Scrum e, à medida que os problemas são identificados e tratados, as melhorias são implantadas a cada sprint.

O Agile DMAIC é composto de três componentes (veja a Figura 1): (i) DMAIC, voltado à melhoria de processos, é utilizado na definição de metas, identificação e análise 
de causas, implantação e controle de melhorias; (ii) CAS, checklist utilizado para coletar a percepção das equipes quanto ao uso de princípios e práticas do Scrum; e (iii) Índice de Medição do Nível do Scrum (IMS), que avalia a adoção das práticas do Scrum.

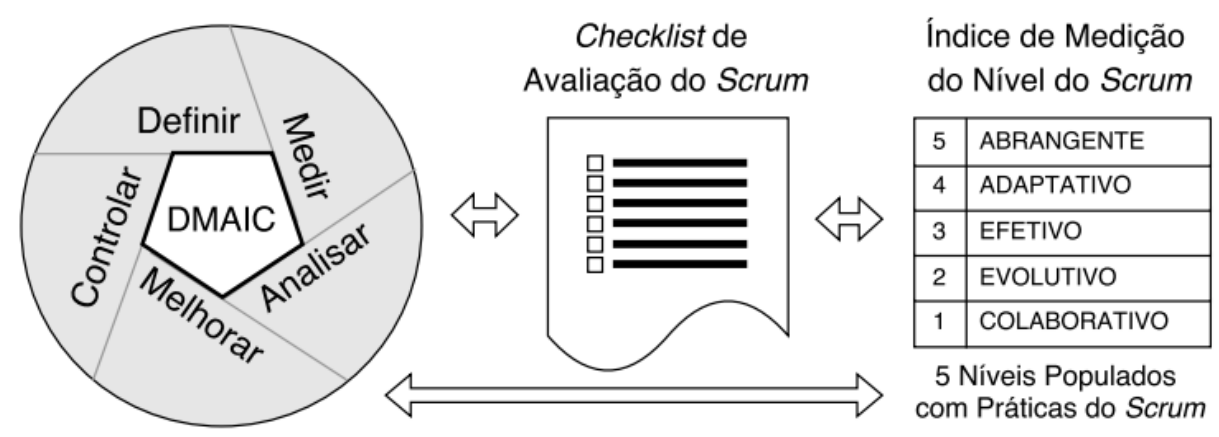

Figura 1. Componentes do Agile DMAIC.

O Agile DMAIC se baseia em coletar periodicamente a percepção da equipe sobre o uso do Scrum e essa percepção é utilizada na identificação de problemas e na classificação do nível do Scrum no projeto. Em seguida, é necessário analisar as causas, definir e implantar ações de melhoria, antecipando os benefícios do Scrum. Mais detalhes sobre o Agile DMAIC podem ser encontrados em [Cunha e Andrade 2014b].

\section{Processo de Elaboração do CAS 2.0}

O processo de elaboração da nova versão do $C A S$ é apresentado na Figura 2. Esse processo é cíclico, possui quatro etapas e inicia com a identificação e seleção de artefatos sobre o Scrum, os quais são os checklists elencados na Seção 2 e o Guia do Scrum.

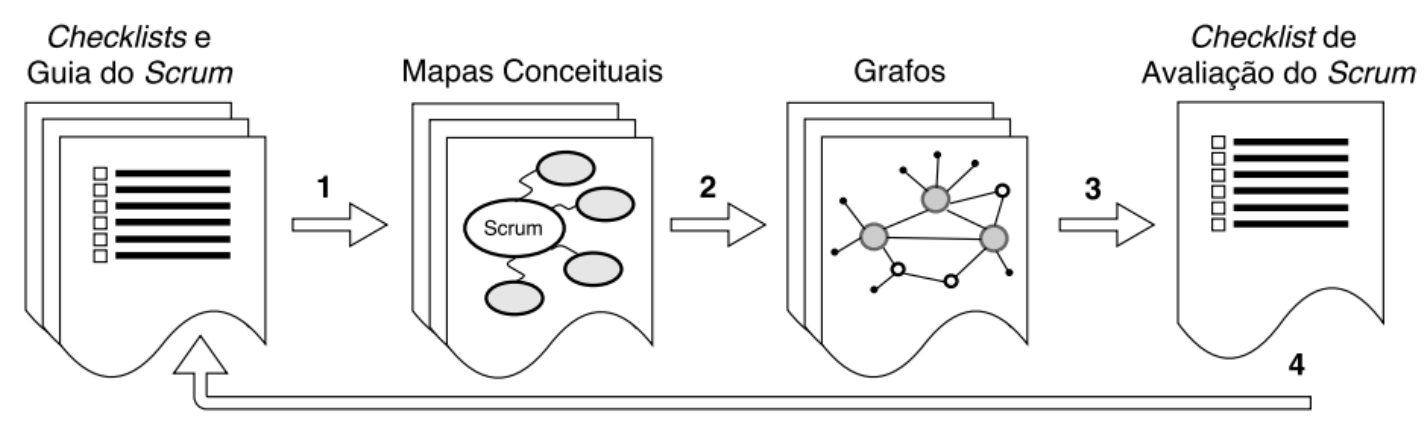

Figura 2. Processo de Elaboração do Checklist de Avaliação do Scrum.

A elaboração de mapas conceituais para os checklists e para o Guia do Scrum é realizada na etapa 1. Esses mapas são representações gráficas da organização de conceitos e regras, em sua estrutura cognitiva, e identificam e relacionam os elementos do Scrum (e.g. artefatos, papéis e eventos). Uma vez que esses mapas podem ser definidos como grafos (com vértices representando os elementos do Scrum e arestas, as relações existentes entre esses elementos), a comparação dos conceitos e regras do Scrum entre os artefatos pode ser vista como um problema de correspondência de grafos, no qual podem ser utilizadas métricas para medição de suas similaridades [De Souza et al. 2008].

A elaboração dos mapas é realizada por especialistas no Scrum e o procedimento manual utilizado é apresentado no Algoritmo 1. Como entradas para esse algoritmo têmse o artefato a ser analisado e os mapas conceituais já criados. O primeiro passo é a análise 
do artefato para identificar as regras e os conceitos sobre o Scrum. Se o elemento identificado é uma regra, então ela é documentada em uma planilha junto às entidades associadas a ela. Se a regra já foi analisada antes, seu identificador é o mesmo atribuído anteriormente; do contrário um novo identificador é gerado. Depois disso, a regra é adicionada ao mapa conceitual. Se o elemento for um conceito, ele é simplesmente adicionado ao mapa conceitual.

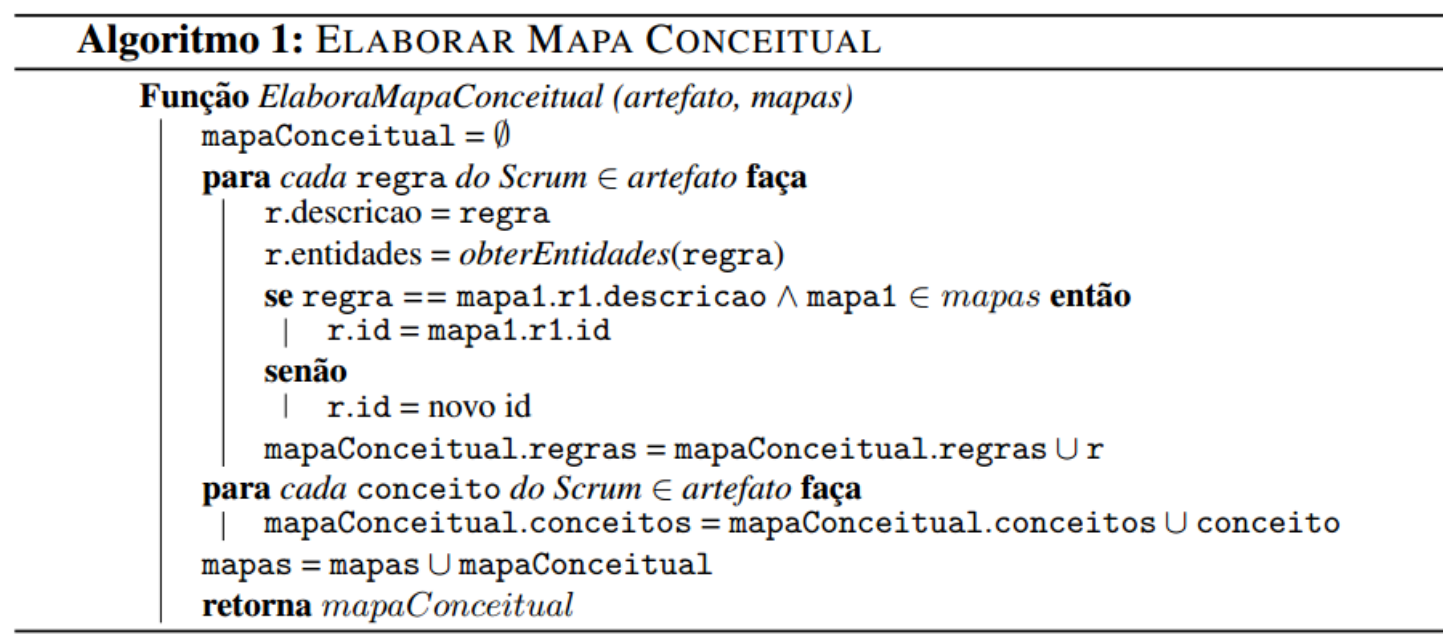

Para cada um dos mapas, documentados em planilhas eletrônicas, são gerados grafos, considerando que: (i) os nós correspondem às entidades; e (ii) as arestas relacionam essas entidades a partir das regras identificadas. A utilização desses grafos é importante para auxiliar na análise e comparação dos artefatos, reduzindo a propensão a falhas durante o processo de elaboração do novo checklist.

Em seguida, são realizadas operações de interseção e subtração dos grafos para uma análise das semelhanças e diferenças entre cada artefato. Essa análise é realizada por especialistas no Scrum.

Um exemplo de um grafo de interseção entre o Guia do Scrum e a nova versão do $C A S$ é apresentado na Figura 3. Os nós representam os elementos do Scrum e as arestas contém os identificadores das regras que relacionam esses elementos. O tamanho do nó é proporcional à quantidade de regras associadas a ele. A nova versão do $C A S$, apresentada na Seção 5, pode ser consultada para a identificação das regras referenciadas nessa figura, considerando que cada identificador corresponde a um item do checklist (e.g., 45 identifica a regra expressa pelo item "[Reunião de Revisão] 45. Feedback é recebido do Product Owner" e no grafo corresponde à aresta entre o Product Owner e a Revisão do Sprint).

O novo checklist é então elaborado ou ajustado, na etapa 3, conforme a análise dos grafos, que objetiva consolidar, a partir dos artefatos, as principais entidades e regras sobre o Scrum.

Esse checklist é então utilizado em projetos reais, na etapa 4, e os feedbacks das equipes possibilitam que ele seja aperfeiçoado. Nessa etapa, alguns itens do checklist podem ser criticados (e.g., quanto à subjetividade e a possibilidade de obter diferentes respostas para um mesmo item). Na prática, essas críticas são solucionadas através das discussões da equipe sobre o resultado consolidado do preenchimento do checklist. 
Nessas discussões, eventuais dúvidas são resolvidas e o entendimento sobre cada item tende a ser alinhado, ou melhorias são sugeridas para o checklist.

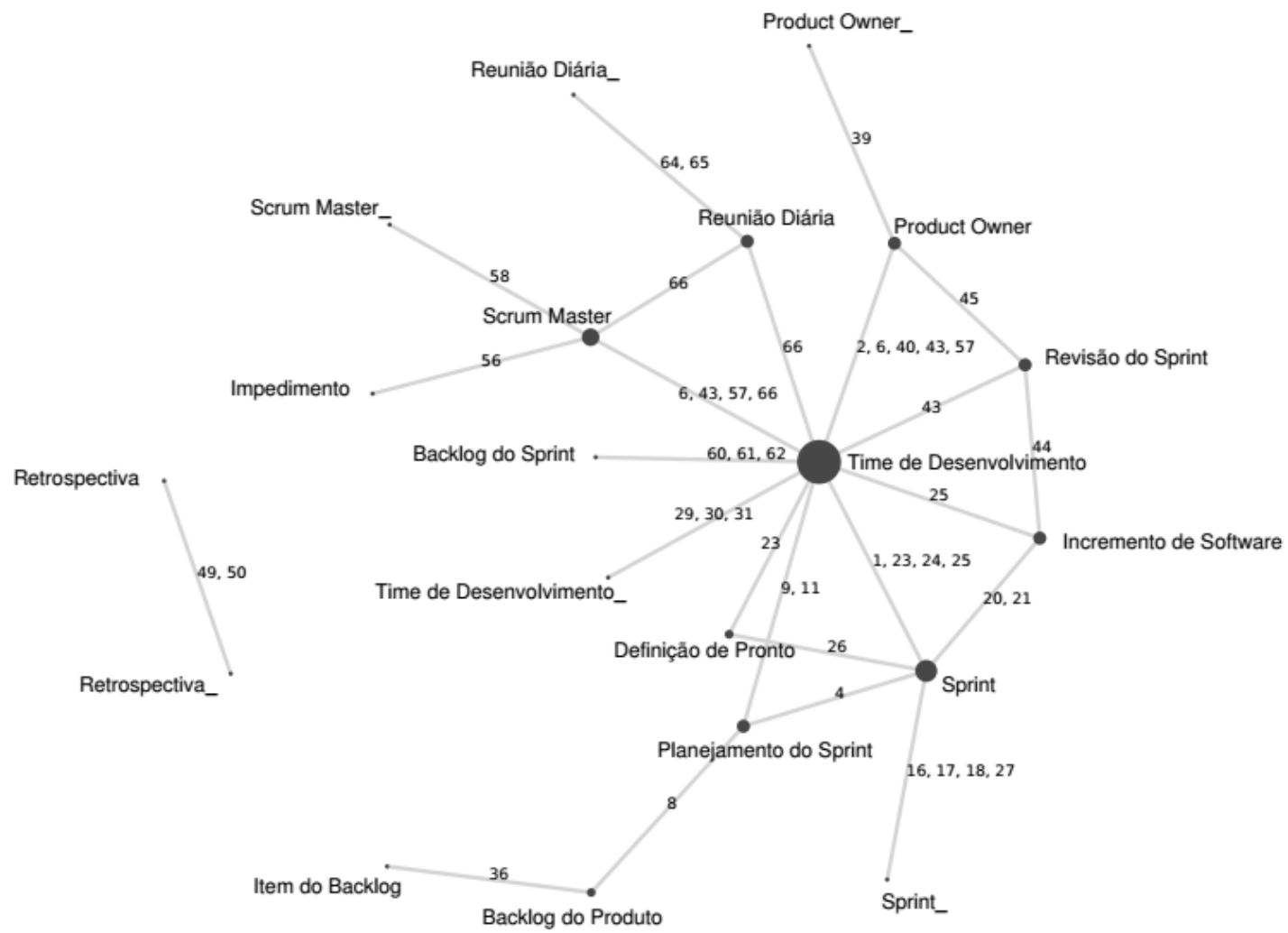

Figura 3. Grafo de Interseção entre o Guia do Scrum e o CAS 2.0.

Após a implantação das melhorias identificadas pelas equipes, o checklist pode compor os artefatos da etapa 1 do processo, além de outras fontes sobre o Scrum, e um novo ciclo de melhoria do CAS pode ser realizado. Os algoritmos utilizados na geração e comparação dos grafos, bem como os artefatos, mapas e os próprios grafos gerados neste estudo estão disponíveis em um repositório no Bitbucket ${ }^{6}$.

\section{Checklist de Avaliação do Scrum (CAS) 2.0}

O CAS $2.0^{7}$ foi desenvolvido a partir do processo descrito na Seção 4, utilizando-se do Guia do Scrum e checklists discutidos na Seção 2, com o intuito de consolidar as principais regras do Scrum, bem como solucionar os pontos de melhoria daqueles checklists.

Essa nova versão do $C A S$ amplia a inspeção do uso de princípios e práticas ágeis para que as equipes melhorem a identificação e tratamento dos problemas no uso do Scrum. Esse checklist continua como um componente do Agile DMAIC, método apresentado na Seção 3, e, embora possa ser utilizado separadamente, recomenda-se que seja aplicado junto ao método.

\footnotetext{
${ }^{6}$ https://bitbucket.org/thiagoferraz/comparacaografos

${ }^{7} \mathrm{http}: / / \mathrm{www}$.agiledmaic.com/home
} 
O CAS 2.0 foi elaborado para ser respondido por toda a equipe Scrum (Product Owner, Scrum Master e Time de Desenvolvimento) a cada iteração, idealmente, momentos antes das reuniões de retrospectiva ou mesmo durante essas reuniões, para que as equipes possam refletir a respeito da evolução do processo ágil, propondo soluções para os principais problemas identificados.

O CAS 2.0 é formado de 70 itens, distribuídos em treze seções, apresentadas na Figura 4. Como exemplo, a seção Planejamento do Sprint possui 11 itens, onde o primeiro item visa identificar se o planejamento é realizado a cada iteração. O CAS 2.0 concentrase em avaliar como são utilizados os eventos e artefatos do Scrum. No entanto, ele também abrange a inspeção do uso dos papéis, fundamentos, valores e princípios dessa metodologia.

O objetivo do CAS 2.0 é avaliar quais princípios e práticas do Scrum estão sendo utilizados nos projetos. Embora alguns itens do checklist indiretamente avaliem a qualidade do produto (e.g., " 25 . Time atento à qualidade do software desenvolvido no sprint" e "44. Software testado é apresentado"), o CAS 2.0 não inspeciona a qualidade com que os princípios e práticas do Scrum são utilizados pelas equipes.

\subsection{Comparativo das Versões do Checklist}

Durante o uso da primeira versão do CAS em projetos reais, as equipes sinalizaram que as avaliações não haviam identificado alguns problemas importantes (e.g., não atendimento dos objetivos do sprint, entregas de software com baixa qualidade, longas reuniões de planejamento e acompanhamento). Dessa forma, havia divergências entre as avaliações com a primeira versão do CAS e os resultados dos projetos.

Nesse cenário, as mudanças realizadas para a nova versão do checklist objetivaram ampliar a inspeção de princípios e práticas do Scrum e suprir as lacunas identificadas. Dentre as alterações, destacam-se as seguintes:

- Objetivos do Sprint - 2 novos itens para inspecionar se, durante o planejamento, os objetivos do sprint foram definidos pelo time e se, ao final do sprint, esses objetivos haviam sido atendidos;

- Qualidade do Incremento de Software - 1 novo item para verificar se o time estava atento a qualidade do software desenvolvido no sprint; e

- Reuniões de Planejamento, Revisão e Acompanhamento Diário - 4 novos itens relacionados à realização e duração das reuniões do Scrum.

Em resumo, a nova versão do checklist, CAS 2.0, manteve a mesma quantidade de seções da primeira versão, com alteração apenas da seção Plano de Lançamentos que, por sugestão das equipes envolvidas no estudo de caso, foi renomeada para Plano de Releases. Já em relação aos itens do checklist, a quantidade de itens aumentou de 65 para 70 e somente 29 dos 65 itens iniciais não sofreram ajustes.

A planilha Comparativo_Ver soes_CAS.xlsx, disponível no repositório do Bitbucket $^{8}$, apresenta um maior detalhamento das mudanças entre as versões do $C A S$.

${ }^{8}$ https://bitbucket.org/thiagoferraz/comparacaografos 
VALORES, PRINCÍPIOS E FUNDAMENTOS

\begin{tabular}{|l|}
\hline$\square$ 01. Time entrega software testado a cada sprint \\
\hline$\square$ 02. Time entrega o que o Product Owner mais prioriza \\
\hline$\square$ 03. Processo está continuamente melhorando \\
\hline
\end{tabular}

\section{PLANEJAMENTO DO SPRINT}

\begin{tabular}{|l|}
\hline$\square$ 04. Há planejamento a cada sprint \\
\hline$\square$ 05. Duração de 8 horas ou menos \\
\hline$\square$ 06. Todo o time participa \\
\hline$\square$ 07. Utiliza Backlog do Produto atualizado \\
$\square$ 08. Product Owner apresenta itens prioritários do backlog \\
\hline$\square$ 09. Somente o Time decide quantos itens de backlog \\
cabem no sprint \\
\hline$\square$ 10. Itens do Backlog do Sprint decompostos em tarefas \\
\hline 11. Somente o Time define e estima tarefas \\
\hline 12. Product Owner disponível quando o Time está estimando \\
\hline$\square$ 13. Time revisa e atualiza a definição de "pronto" \\
$\square$ 14. Objetivos do sprint claramente definidos \\
\hline
\end{tabular}

\section{SPRINT}

\begin{tabular}{|l|}
\hline$\square$ 15. Há iterações definidas \\
$\square$ 16. Duração de 4 semanas ou menos \\
$\square$ 17. Datas de início e fim do sprint respeitadas \\
$\square$ 18. Estimativas atualizadas durante o sprint \\
$\square$ 19. Testes de aceitação realizados durante o sprint \\
$\square$ 20. Software desenvolvido no sprint testado durante o \\
próprio sprint \\
$\square$ 21. Software é implantado em ambiente para validação do \\
Product Owner \\
$\square$ 22. Alocação de tarefas realizada somente pelo Time \\
$\square$ 23. Time conhece e respeita a definição de "pronto" do sprint \\
\hline$\square$ 24. Time não é interrompido nem controlado de fora \\
$\square$ 25. Time atento à qualidade do software desenvolvido no \\
sprint \\
\hline$\square$ 26. Velocidade do sprint é medida considerando apenas os \\
itens "prontos" \\
\hline$\square$ 27. Todos os objetivos do sprint foram alcançados \\
\hline
\end{tabular}

\section{TIME DE DESENVOLVIMENTO}

\begin{tabular}{|l|}
\hline$\square$ 28. Costuma entregar o que foi prometido \\
\hline$\square$ 29. Possui as competências necessárias \\
\hline$\square$ 30. Integrantes não estão dedicados a papéis específicos \\
\hline$\square$ 31. Possui de 3 a 9 integrantes \\
\hline$\square$ 32. Conhece os principais impedimentos \\
\hline
\end{tabular}

\section{BACKLOG DO PRODUTO}

\begin{tabular}{|l|}
\hline$\square$ 33. Há um backlog do produto \\
\hline$\square$ 34. Priorizado por valor de negócio \\
\hline$\square$ 35. Time compreende os itens de backlog \\
\hline$\square$ 36. Itens prioritários grandes decompostos em menores \\
\hline 37. Acessivel a todo o time \\
\hline
\end{tabular}

PRODUCT OWNER

\begin{tabular}{|l|}
\hline$\square$ 38. Claramente definido \\
\hline$\square$ 39. Tem conhecimento e autoridade para priorizar \\
\hline$\square$ 40. Interage com todo o time \\
\hline
\end{tabular}

\section{REUNIÃO DE REVISÃO}

\begin{tabular}{|l|}
\hline$\square$ 41. Time realiza a reunião de revisão a cada sprint \\
\hline$\square$ 42. Duração de 4 horas ou menos \\
\hline$\square$ 43. Todo o time participa \\
\hline$\square$ 44. Software testado é apresentado \\
$\square$ 45. Feedback é recebido do Product Owner \\
\hline
\end{tabular}

\section{RETROSPECTIVA}

\begin{tabular}{|l|}
\hline$\square$ 46. Há retrospectiva ao final do sprint \\
\hline$\square$ 47. Duração de 3 horas ou menos \\
\hline$\square$ 48. Time e Scrum Master participam \\
\hline 49. Resulta em propostas de melhorias concretas \\
\hline$\square$ 50. Algumas propostas são implementadas \\
\hline
\end{tabular}

\section{PLANO DE RELEASES}

$\square$ 51. Product Owner tem plano de releases com base no Backlog do Produto

$\square$ 52. Product Owner usa velocidade do Time para planejar releases

$\square$ 53. Acompanhado e atualizado a cada sprint pelo Product Owner

$\square$ 54. Acessivel a todo o time

\section{SCRUM MASTER}

$\square$ 55. Claramente definido

$\square$ 56. Focado em remover impedimentos

$\square$ 57. Interage com todo o time

$\square$ 58. Conhece e dissemina os princípios e práticas do Scrum

\section{BACKLOG DO SPRINT}

$\square$ 59. Time utiliza backlog do sprint

$\square$ 60. Pertence exclusivamente ao Time

$\square$ 61. Acompanhado e atualizado diariamente pelo Time

$\square$ 62. Acessivel a todo o time

\section{REUNIÃo DIÁRIA}

\begin{tabular}{|l|}
\hline$\square$ 63. Há reuniōes diárias \\
\hline$\square$ 64. Duração de 15 minutos ou menos \\
$\square$ 65. Ocorre no mesmo horário e local \\
\hline$\square$ 66. Time e Scrum Master participam \\
\hline$\square$ 67. Problemas e impedimentos são informados \\
\hline
\end{tabular}

\section{GRÁFICO DE BURNDOWN}

\begin{tabular}{|l|}
\hline$\square$ 68. Time utiliza um gráfico de Burndown \\
\hline$\square$ 69. Acompanhado e atualizado diariamente pelo Time \\
\hline$\square$ 70. Acessivel a todo o time \\
\hline
\end{tabular}

Figura 4. CAS 2.0 - Checklist de Avaliação do Scrum. 


\subsection{Sobre a Automação do Checklist}

A automação do CAS 2.0 foi realizada a partir da plataforma Google Sites ${ }^{9}$ e está disponível online através do endereço http://www.agiledmaic.com/. O Google Sites possibilita a criação rápida de sites e foi selecionado neste estudo por ser gratuito, de fácil aprendizado e uso, e por fornecer os componentes necessários para a automação.

A automação do CAS 2.0 pode ser dividida em três funcionalidades: (i) Preencher o CAS: implementação realizada com o Google Forms, integrado ao Google Sites; (ii) Realizar os cálculos de abrangência e nível de maturidade no uso do Scrum, os quais são realizados de acordo com a especificação do método Agile DMAIC, a partir das fórmulas das planilhas e dados do preenchimento das equipes; e (iii) Consolidar os resultados da equipe e apresentá-los em tempo real, e vale ressaltar que nessa funcionalidade foram utilizados os componentes gráficos Google Charts e o Awesome Table.

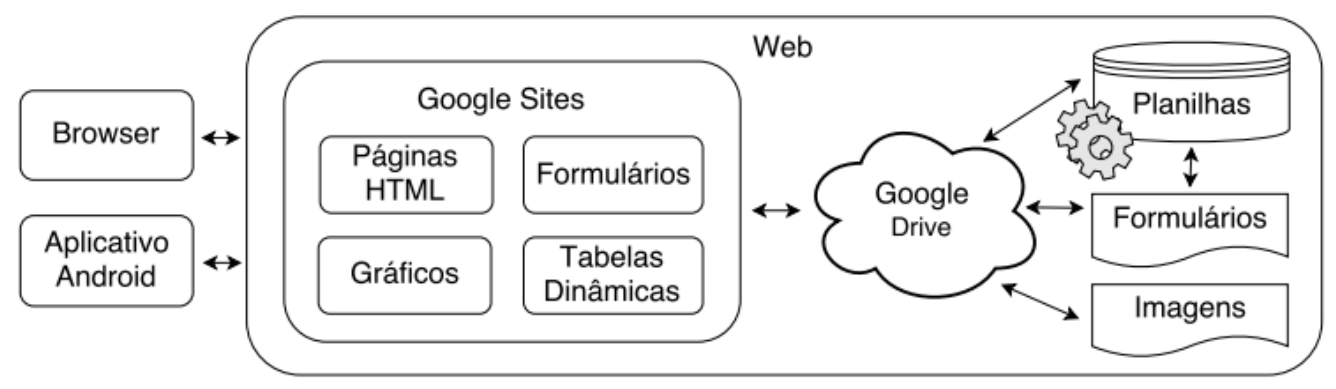

Figura 5. Arquitetura da Automação do CAS 2.0.

A arquitetura da automação do CAS 2.0 é apresentada na Figura 5. O CAS 2.0 pode ser acessado na Web através de um browser ou pelo aplicativo Android disponível no Google Play ${ }^{10}$. O Google Sites fornece uma variedade de componentes para a criação dos sites. Os principais componentes utilizados foram as páginas $H T M L$, formulários, gráficos e um componente para geração de tabelas dinâmicas, Awesome Table. Esses componentes, por sua vez, possuem integração com as planilhas, formulários e imagens armazenados e gerenciados na nuvem pelo Google Drive ${ }^{11}$.

\section{Aplicando o CAS 2.0 em Projetos Reais}

Esta seção apresenta uma avaliação qualitativa da aplicação do $C A S 2.0$ em projetos reais, na qual avalia-se as informações e também as traduz em números para serem classificadas e analisadas [Severino 2007].

\subsection{Objetivo da Avaliação do CAS 2.0}

O objetivo da avaliação foi definido com base no template de definição de objetivos do Goal-Question-Metric (GQM) [Basili et al. 1995]. O objetivo do estudo de caso é analisar o $C A S 2.0$, com a finalidade de avaliação, no que diz respeito a sua estrutura e utilização, do ponto de vista dos papéis do Scrum, no contexto de projetos de desenvolvimento de software com o Scrum. Para atingir esse objetivo, foram definidas três questões de análise e cinco medidas, conforme são apresentadas na Figura 6 e descritas a seguir:

\footnotetext{
${ }^{9} \mathrm{https}: / /$ apps.google.com/intx/en/products/

${ }^{10} \mathrm{https}$ ///play.google.com/store/apps/details?id=com.ah2 .apps.agile2us

${ }^{11} \mathrm{https} / / / \mathrm{www} . \mathrm{google} . \mathrm{com} /$ drive/
} 
- (M1) Clareza e objetividade - Avalia se o checklist usa uma linguagem simples e direta, sendo breve e objetivo na identificação do essencial sobre o uso do Scrum;

- (M2) Ordem e organização das seções e itens - Avalia se os itens do checklist estão organizados em uma ordem lógica, numerados e agrupados por similaridades;

- (M3) Quantidade de seções e itens - Avalia o checklist quanto ao seu tamanho a partir da quantidade de seções e itens;

- (M4) Tempo de preenchimento - Avalia o tempo de preenchimento do checklist; e

- (M5) Importância para identificar problemas no uso do Scrum - Avalia o checklist quanto a sua importância na identificação de problemas no uso do Scrum.

Questões

Medidas

\begin{tabular}{|l|l|l|}
\hline $\begin{array}{l}\text { Q1 } \\
\text { Objetivo }\end{array}$ & $\begin{array}{l}\text { O CAS 2.0 é facilmente } \\
\text { entendido pelo time Scrum? }\end{array}$ \\
\hline CAS 2.0 & $\begin{array}{l}\text { O CAS 2.0 permite ser } \\
\text { rapidamente preenchido } \\
\text { pelo time Scrum? }\end{array}$ \\
\hline Q3 & $\begin{array}{l}\text { O CAS 2.0 possibilita } \\
\text { identificar problemas no } \\
\text { uso do Scrum? }\end{array}$ \\
\hline
\end{tabular}

Figura 6. Relacionamento entre as questões e medidas da avaliação.

Sobre o método de coleta, as informações necessárias às medidas foram obtidas com as equipes dos projetos, a partir de um questionário ${ }^{12}$ aplicado via Web. Esse questionário contém 4 perguntas sobre o perfil dos respondentes, 5 sobre as medidas e 1 pergunta aberta para sugestões de melhorias sobre o checklist. Nas questões relacionadas às medidas, os participantes deveriam indicar suas opiniões a partir de um dos seguintes valores: Péssimo, Ruim, Regular, Bom ou Excelente. O resultado final de cada medida foi obtido aplicando-se a moda dos valores indicados pelos respondentes.

\subsection{Projetos Reais do Estudo de Caso}

Os projetos selecionados para o estudo de caso foram desenvolvidos em duas instituições públicas e uma privada, que utilizaram o CAS 2.0 durante a adoção do Scrum ou na melhoria de sua utilização. Um resumo desses projetos, com informações obtidas dos líderes através de um questionário ${ }^{13}$ aplicado via $W e b$, é apresentado na Tabela 2. Por motivo de confidencialidade, a razão social das instituições envolvidas, bem como informações mais detalhadas sobre os projetos não são apresentadas.

As instituições A e C são públicas, enquanto a $\mathrm{B}$ é privada. Todas as equipes envolvidas são formadas, em sua maioria, por pesquisadores e desenvolvedores de áreas da computação e engenharia, e a maior parte dos integrantes possui uma alocação integral aos projetos. Na Tabela 2, há projetos de Desenvolvimento de Software (D) e de Pesquisa e Desenvolvimento (P\&D). A Duração (Meses) consiste na duração planejada dos projetos e a Equipe representa o tamanho das equipes, considerando o Product Owner, Scrum Master e o Time de Desenvolvimento. A Complexidade dos projetos está relacionada ao uso simultâneo de várias tecnologias, ao elevado número de envolvidos e à necessidade de conhecimentos técnicos especializados.

\footnotetext{
${ }^{12} \mathrm{http}: / / \mathrm{www}$.agiledmaic.com/home/feedback-do-checklist

${ }^{13} \mathrm{http}$ ///www.agiledmaic.com/projetos/novo-projeto
} 
Tabela 2. Projetos do Estudo de Caso.

\begin{tabular}{|l|c|c|c|c|c|}
\hline Projeto & Instituição & Tipo de Projeto & Duração (Meses) & Equipe & Complexidade \\
\hline PID_1977 & A & D & 10 & 10 & Média \\
\hline PID_2103 & A & D & 3 & 5 & Baixa \\
\hline PID_2131 & A & D & 3 & 9 & Média \\
\hline PID_2139 & A & D & 4 & 10 & Média \\
\hline PID_FV6 & A & D & 5 & 10 & Alta \\
\hline PID_PP & A & D & 6 & 5 & Média \\
\hline PID_PR & A & D & 6 & 6 & Média \\
\hline Mobile App & B & D & 6 & 9 & Média \\
\hline Tools - App1 & B & P\&D & 6 & 8 & Alta \\
\hline Tools - App2 & B & P\&D & 6 & 6 & Média \\
\hline Tools - App3 & B & P\&D & 6 & 6 & Média \\
\hline Tools - App4 & B & P\&D & 6 & 7 & Alta \\
\hline Tools - App5 & B & P\&D & 6 & 3 & Média \\
\hline Tools - App6 & B & P\&D & 3 & 5 & Alta \\
\hline SGPR & C & D & 7 & 10 & Média \\
\hline
\end{tabular}

\subsection{Resultados e Discussão}

As informações apresentadas nesta seção foram obtidas com as equipes dos projetos do estudo de caso. No total, 53 pessoas participaram da avaliação do $C A S 2.0$, de forma espontânea e anônima. Sobre os respondentes, 75,5\% (40) deles eram da instituição A, $13,2 \%$ (7) da B e $11,3 \%$ (6) da C.

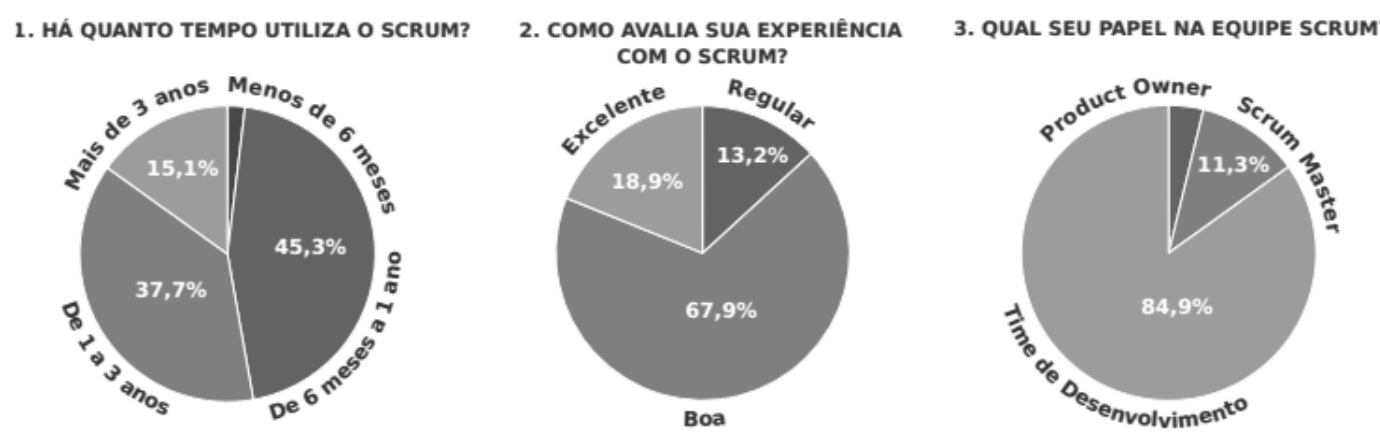

Figura 7. Tempo, Experiência e Papel dos Respondentes do Estudo de Caso.

Os gráficos da Figura 7 apresentam, respectivamente, o tempo, a experiência e o papel dos respondentes com o Scrum. Quanto ao uso do Scrum, 98,1\% dos respondentes já tinham mais de seis meses de experiência e, além disso, 86,8\% afirmaram ter uma experiência boa ou excelente com o Scrum. Dessa forma, boa parte dos participantes conhecia bem os conceitos e práticas utilizados. Sobre o papel dos respondentes, houve a participação dos três papéis do Scrum, sendo 3,8\% (2) deles Product Owner, 11,3\% (6) eram Scrum Masters e 84,9\% (45) eram do Time de Desenvolvimento.

Em se tratando do $C A S$, todos os participantes utilizaram o checklist uma ou mais vezes, e $52,8 \%$ deles, seis ou mais vezes. No que diz respeito ao conteúdo do $C A S$, os resultados da avaliação das medidas foram positivos (veja a Figura 8):

- (M1) 86,8\% avaliaram a clareza e a objetividade do CAS como Bom ou Excelente. O resultado da moda para M1 foi Bom $(54,7 \%)$;

- (M2) 90,6\% consideraram a ordem e a organização dos itens e seções como Bom ou Excelente. O resultado da moda para M2 foi Bom (56,6 \%); 
- (M3) 81,1\% avaliaram o tamanho do checklist como Bom ou Excelente. O resultado da moda para M3 foi $\operatorname{Bom}(58,5 \%)$;

- (M4) 86,8\% avaliaram o tempo de preenchimento como Bom ou Excelente. O resultado da moda para M4 foi Bom (56,6\%); e

- (M5) $88,7 \%$ acreditam que o CAS é importante para identificar problemas no uso do Scrum, avaliando o checklist como Bom ou Excelente. O resultado da moda para M5 foi $\operatorname{Bom}(54,7 \%)$.

1. QUANTAS VEZES UTILIZOU O CAS?

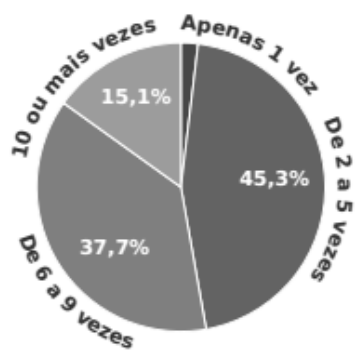

4. COMO AVALIA? [ORDEM E ORGANIZAÇĀO DAS SEÇŌES E ITENS]

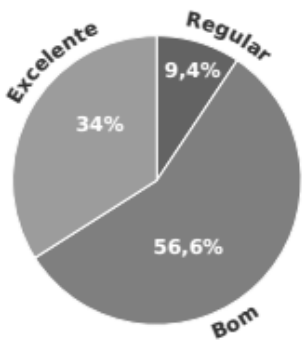

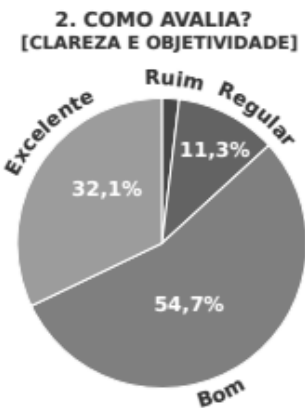

5. COMO AVALIA? [TEMPO DE PREENCHIMENTO]

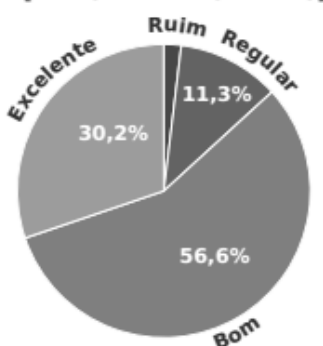

3. COMO AVALIA? [QUANTIDADE DE SEÇŌES E ITENS]

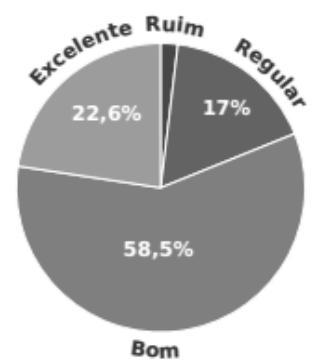

6. COMO AVALIA?

[IMPORTÃNCIA PARA IDENTIFICAR PROBLEMAS]

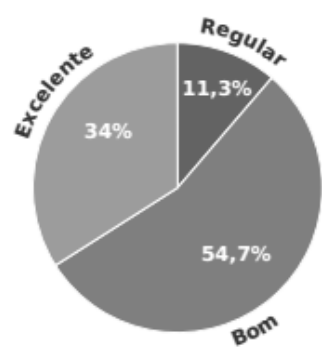

Figura 8. Feedback das Equipes sobre a Nova Versão do CAS.

Com base nos valores das medidas, foi possível responder às questões definidas: (Q1) O CAS 2.0 é de fácil compreensão, pois ele é claro e objetivo $(\mathrm{M} 1=$ Bom $)$, além de possuir uma boa organização dos itens e seções $(\mathrm{M} 2=$ Bom $)$; $(\mathbf{Q 2})$ O preenchimento do $C A S 2.0$ pode ser considerado rápido, uma vez que ele é de fácil compreensão, tem uma quantidade satisfatória de itens e seções $(\mathrm{M} 3=B o m)$ e tem um tempo de preenchimento satisfatório (M4 = Bom); e (Q3) O CAS 2.0 é de fácil entendimento $(\mathrm{M} 1=$ bom) e auxilia na identificação de problemas no uso do Scrum $($ M5 $=$ Bom). Outras análises (e.g., uma análise por papéis, experiência no Scrum) podem ser encontradas na $W e b^{14}$.

Além disso, como no questionário havia uma questão aberta para sugestões de melhoria, foi possível obter outros feedbacks a serem utilizados para a evolução do checklist. Os principais comentários são descritos a seguir: (i) "Alguns itens possuem certa relação e isso poderia ser utilizado para ocultar (em tempo real, no preenchimento) os itens desnecessários, diminuindo o esforço do usuário e o tempo de preenchimento"; (ii) "Diminuir a quantidade de seções ou deixá-las de acordo com o nível do time ou do sprint a ser avaliado"; (iii) "Adicionar uma descrição do que se busca inspecionar com cada item ou seção"; e (iv) "Permitir que apenas a quantidade informada de integrantes do time preencha o checklist, de forma a não alterar a avaliação final". Conforme supracitado, a maioria dos feedbacks visam a otimização do preenchimento do $C A S$.

\footnotetext{
${ }^{14}$ http://www .agiledmaic.com/feedback-sobre-o-checklist
} 
Outras preocupações dos respondentes estão relacionadas à segurança e usabilidade do checklist.

\section{Conclusões}

Para que os projetos usufruam dos principais benefícios do Scrum, como a melhoria na produtividade e qualidade dos produtos desenvolvidos, sua adoção e uso precisam ser adequadamente gerenciados para uma evolução contínua e controlada do processo ágil. Nesse cenário, para avaliar o uso de seus princípios e práticas, uma nova versão do Checklist de Avaliação do Scrum (CAS) foi proposta neste artigo, elaborada com base no Guia do Scrum e em mais sete checklists. A principal vantagem dessa nova versão, CAS 2.0, é que, além de melhorar a inspeção de princípios e práticas do Scrum, sua automação permite consolidar a visão dos integrantes da equipe sobre o uso do Scrum e fornecer resultados em tempo real.

Para elaborar o CAS 2.0, foi definido um processo com quatro etapas: (i) identificação e seleção de artefatos sobre o Scrum; (ii) criação dos mapas conceituais com base nas regras e conceitos identificados; (iii) geração de grafos a partir desses mapas; e (iv) consolidação da nova versão do checklist através da análise das operações de subtração e interseção dos grafos. Uma vez que esse processo pode ser utilizado na melhoria do $C A S$, acredita-se que ele pode ser usado para melhorar outros checklists.

Esse checklist foi aplicado em quinze projetos reais, durante um ano, envolvendo três instituições. Para coletar o feedback dos usuários, foi elaborado um questionário online, o qual obteve 53 respondentes. De maneira geral, os resultados foram positivos, pois, em todos os critérios avaliados (objetividade, tamanho, organização, tempo de preenchimento e importância para identificar problemas no uso do Scrum), a aprovação da nova versão do $C A S$ foi superior a $80 \%$.

Como trabalhos futuros, pretende-se: (i) realizar uma análise comparativa mais aprofundada dos checklists de avaliação do Scrum, com base na Engenharia de Software Experimental; (ii) traduzir o CAS 2.0 para outros idiomas; (iii) aumentar a segurança quanto ao seu preenchimento online; e (iv) implementar mecanismos de adaptação do checklist para diferentes contextos (e.g., experiência e maturidade da equipe com o Scrum e metas de melhoria no uso de princípios e práticas ágeis).

\section{Referências}

Basili, V. R., Caldiera, G., e Rombach, H. D. (1995). Goal question metric paradigm. In Encyclopedia of Software Engineering, pages 527-532. John Wiley Sons.

Cunha, T. F. V. d. e Andrade, R. M. C. (2014a). Agile DMAIC: Um método para avaliar e melhorar o uso do Scrum em projetos de software. In XIII Simpósio Brasileiro de Qualidade de Software (SBQS), pages 121-135.

Cunha, T. F. V.d. e Andrade, R. M. C. (2014b). SLeSS 2.0: uma evolução da abordagem de integração do Scrum e lean six sigma para aplicações móveis.

De Souza, F., Boeres, M., Cury, D., De Menezes, C., e Carlesso, G. (2008). An approach to comparison of concept maps represented by graphs.

Gloger, B. (2012). Infoq scrum checklist. Disponível em: https://www.infoq.com/ minibooks/scrum-checklists. Acesso em: 23 de Maio de 2016. 
Jalali, S., Wohlin, C., e Angelis, L. (2014). Investigating the applicability of agility assessment surveys: A case study. Journal of Systems and Software, 98:172 - 190.

Khelladi, D.-E., Bendraou, R., Baarir, S., Laurent, Y., e Gervais, M.-P. (2015). A framework to formally verify conformance of a software process to a software method. In Proceedings of the 30th Annual ACM Symposium on Applied Computing, SAC '15, pages 1518-1525, New York, NY, USA. ACM.

Kniberg, H. (2009). Scrum Checklist - version 2.0. Disponível em: http://blog.crisp. se/2009/08/14/henrikkniberg/1250265360000. Acesso em: 04 de Julho de 2016.

Lomonaco, F. e Lapointe, C. (2013). Scrum Adherence Index. Disponível em: https://goo.gl/VdaLpp. Acesso em: 23 de Maio de 2016.

Maharana, A. (2015). A Scrum Master's Checklist. Disponível em: https://www. linkedin.com/pulse/scrum-masters-checklist-anand-maharana. Acesso em: 23 de Maio de 2016.

Melo, C. O., Santos, V. A., Corbucci, H., Katayama, E., Goldman, A., e Kon, F. (2012). Métodos ágeis no Brasil: estado da prática em times e organizações. Technical Report RTMAC201203.

Park, J. S., McMahon, P.E., e Myburgh, B. (2016). Scrum powered by essence. SIGSOFT Softw. Eng. Notes, 41(1):1-8.

Perkusich, M., Soares, G., Almeida, H., e Perkusich, A. (2015). A procedure to detect problems of processes in software development projects using Bayesian networks. Expert Systems with Applications, 42(1):437 - 450.

Radujkovic, M., Vukomanovic, M., Wagner, R., Tenera, A., e Pinto, L. C. (2014). A Lean Six Sigma (LSS) project management improvement model. Procedia - Social and Behavioral Sciences, 119:912 - 920.

Schwaber, K. (2007). The Enterprise and Scrum. Microsoft Press.

Severino, A. J. (2007). Metodologia do trabalho científico. Cortez,São Paulo,23 edition.

Sutherland, J. e Schwaber, K. (2016). Guia do Scrum. Disponível em:http://www.scrumguides.org/docs/scrumguide/v2016/2016-Scrum-GuidePortuguese-Brazilian.pdf. Acesso em: 04 de Agosto de 2016.

TPC (2016). Agile/Scrum Check List - Total Programme Control Ltd. Disponível em: http://www.totalprogrammecontrol.com/scrum.php. Acesso em: 23 de Maio de 2016.

VersionOne (2016). 10th annual state of agile development survey results. Disponível em: https://versionone.com/pdf/VersionOne-10th-Annual-Stateof-Agile-Report.pdf. Acesso em: 04 de Julho de 2016.

Vode, B. e Sutherland, J. (2008). Scrum but test. Disponível em: http://antoine. vernois.net/scrumbut/?page=test\&lang=en. Acesso em: 27 de Junho de 2015 .

Zazworka, N., Stapel, K., Knauss, E., Shull, F., Basili, V. R., e Schneider, K. (2010). Are developers complying with the process: An XP study. In Proceedings of the 2010 ACM-IEEE International Symposium on Empirical Software Engineering and Measurement, ESEM '10, pages 14:1-14:10, New York, NY, USA. ACM. 